\title{
Towards transparency
}

\section{Sharing data is key for efficient scientific progress. More open code would be beneficial too.}

Science thrives on reproducibility. In the politicized realm of the climate sciences, for example, it has long been good practice to have three independent reconstructions of the global temperature record ${ }^{1-3}$. And still, when a fourth one appeared ${ }^{4}$, largely confirmatory of the existing three, it was greeted with a media storm - mainly because the authors had emphasized their independence of the entire climate science community in the run-up to the announcement of their work ${ }^{5}$.

Two ingredients are essential for reproducibility in any field in science: full disclosure of the methods used to obtain and analyse data, and availability of the data that went into and came out of the analysis. Data disclosure has long been one of our policies. To help with the second aspect, the Nature family of journals is now introducing a policy on computer code: we strongly encourage sharing of code and consider it best practice wherever possible. When code is central to obtaining the results presented in a paper, we now require a statement about code availability in the Methods section of our papers, which must include information on how to obtain code and a description of any limitations to its availability.

Sharing code is not always simple. As argued in a Commentary on page 779 in this issue, complex code such as that used in global climate models cannot easily be used by others in a meaningful way. In general, substantial effort is required to make a complex piece of software run on a different machine, and in some cases, it may not be possible. There can also be other technical, legal and commercial restrictions to code sharing. In recognition of these difficulties, Nature journals do not mandate that code be made fully available, and instead only require that the underlying equations be published in sufficient detail to allow reproducibility ${ }^{6}$. But we strongly encourage authors to make their code available where possible, both to make it as clear as possible to readers exactly what was done, and to allow others to build on their work.

Issues around data sharing have been in the spotlight longer. The benefits of sharing data, not only for scientific progress, but also for the careers of individuals, are slowly being recognized. Nevertheless, more incentives are needed to encourage researchers to transfer their private data archives to public repositories together with all the necessary metadata, as suggested in a Commentary on page 778 in this issue.

Making fully annotated, high-quality data publicly available for re-use already brings recognition, citations and professional collaborations to individuals, and much faster progress to science. Many of these benefits could equally apply to code sharing, once it is established as best practice, and fully recognized as part of the scientific endeavour. We are hoping that our code-sharing policy will pave the way.

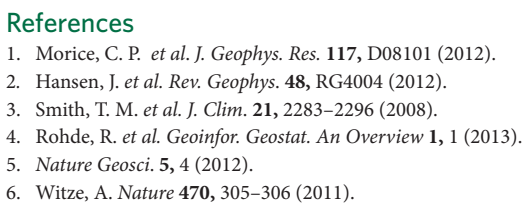

\section{Acquired risk}

\section{Wealth in a country typically protects against earthquake damage. The same cannot always be said for wealth of individuals.}

Earthquakes affect rich and poor countries alike, yet the poorest countries and communities typically suffer more profoundly. The disproportionate vulnerability of the world's most poorly developed nations to earthquake activity was laid bare by the Haiti earthquake in January 2010. The magnitude-7 quake occurred close to Haiti's capital city, Port-au-Prince, with a population of about 940,000; estimates of the death toll from the quake range from 100,000 to 300,000 . For comparison, the magnitude- 6.9 earthquake that struck the 1.5-million-inhabitant city of Kobe, Japan in 1995 caused less than 6,500 fatalities. Of course, no two earthquakes are the same, but the difference in impact is striking: wealth, and hence preparedness, in a country can help protect greatly from earthquake damage.

Wealth of individuals does not necessarily provide such a buffer, as is highlighted by an examination of neighbourhood compositions in Los Angeles, California. Here, comparing the distribution of social groups with the locations of known active faults did not reveal an exposure of socially vulnerable communities to the greatest seismic hazards, as expected (N. A. Toké et al., Earth's Future 2, 440-457; 2014). Instead, the houses owned by the richest residents of Los Angeles turned out to be closest to some of the city's most active faults.

The cause could be traced to the AlquistPriolo Earthquake Fault Zoning Act, which prohibits the construction of new houses in California close to the surface trace of known active faults. Intended to create buffer zones around areas of high seismic risk, the building restriction led to urban developments with much open green space and park land. The seismic hazard in these areas is known, but the risk seems to have been outweighed by the amenities of living next to park land.

Social status and vulnerability to seismic shaking was also found to be less straightforward than expected in the Haiti earthquake (M. K. Lindell, Nature Geosci. 3, 739-740; 2010). Middle-class dwellings, rather than the poorest neighbourhoods, were damaged most severely. Expensive houses that were designed to withstand earthquakes performed well. But, so too did the shanty houses. The lightness of these small huts, made of wood with corrugated metal roofs, made them resistant to shaking. In contrast, many of the concrete-based middle-class houses collapsed during the quake.

Poverty and vulnerability to natural disasters are undeniably linked at the national scale. But at the individual level, some people choose to ignore unlikely, but devastating events and buy themselves into risk. 